121 INCIDENTAL EXTRA-CARDIAC FINDINGS ON CLINICAL CMR; A COMPARISON OF 3 HASTE TECHNIQUES

doi:10.1136/heartjnl-2011-300198.121

${ }^{1} \mathrm{R}$ B Irwin, ${ }^{2} \mathrm{~T}$ Newton, ${ }^{3} \mathrm{C}$ Peebles, ${ }^{4} \mathrm{~A}$ Borg, ${ }^{5} \mathrm{D}$ Clark, ${ }^{4} \mathrm{C}$ Miller, ${ }^{6} \mathrm{~N}$ Abidin, ${ }^{4} \mathrm{M}$ Greaves, ${ }^{4} \mathrm{M}$ Schmitt. 'Wythenshawe Hospital, Manchester, UK; ${ }^{2}$ Royal Blackburn Infirmary, Blackburn, UK; ${ }^{3}$ Southampton General Hospital, Southampton, UK; ${ }^{4}$ Wythenshawe Hospital, University Hospitals of South Manchester NHS Trust, Manchester, UK; ${ }^{5}$ Alliance Medical, Wythenshawe Hospital CME unit, Manchester, UK; ${ }^{6}$ Salford Royal Hospital, Salford, UK

Introduction Cardiac magnetic resonance (CMR) is an increasingly important imaging modality, which by necessity incorporates a large field of view. Both "localiser" and multiple slice half-fourier spin echo (eg, HASTE) sequences provide coverage of the thorax and upper abdomen. Such imaging may reveal hitherto unexpected incidental extra-cardiac findings (IEF). First we sought to assess the frequency of IEF found on clinically indicated CMR scans. Second we compared the 3 clinically used HASTE acquisition protocols in this context. Lastly we determined the impact of the 3 different protocols on acquisition time and image quality.

Methods Three subsequent groups of 238 patients (714 patients in total), all referred for clinically indicated CMR, were scanned with either breath-hold (BH) HASTE (Group 1), free breathing (FB) HASTE (Group 2) or diaphragmatic navigated (NAV) HASTE (Group 3). Additionally "localiser" sequences performed in 3 orthogonal planes were analysed. All 714 clinical reports were reviewed regarding the presence of IEF. These were categorised as either minor, or major if recommendations for further investigation, follow-up, and/or clinical correlation were made. Finally, to determine the impact of each HASTE protocol on acquisition time and image quality, an additional cohort of 15 patients underwent 3 protocols back to back in a random fashion. The length of each acquisition was timed and image quality was reviewed and scored externally.

Results A total of 180 IEF were found in 162 (22.7\%) out of 714 patients. There was no significant difference in frequency of IEF between the 3 HASTE groups. Out of 180 IEF, 88 were considered minor and 92 major findings. Of the latter, 8 (1.1\%) were considered highly significant. These included one bronchoalveolar carcinoma stage $1 \mathrm{~B}$ requiring lobectomy, 2 cases of florid sarcoidosis in patients presenting with VT and "structurally normal hearts" on echocardiography, one case of pulmonary aspergillosis, 2 cases of advanced pulmonary fibrosis, one ascending thoracic aortic aneurysm and a case of iatrogenic liver haemorrhage following placement of a pericardial drain. FB HASTE acquisition $(69 \pm 2.5 \mathrm{~s})$ was significantly faster than BH (105 $\pm 3.8 \mathrm{~s})$ and NAV (121 $\pm 2.7 \mathrm{~s})$, $\mathrm{p}<0.001$, but also produced the lowest image quality on a 5 point scale; $3.5(\mathrm{FB})$ vs $3.9(\mathrm{BH})$ vs $3.8(\mathrm{NAV}), \mathrm{p}=0.08$.

Conclusion Overall, IEF are common and lead to follow on investigations in a substantial minority of cases. However, the overall incidence of highly significant findings in the current study was low $(\sim 1 \%)$. There was no difference in the frequency of incidental extracardiac findings between the 3 HASTE protocols. While the free breathing HASTE technique is statistically significantly faster than breath hold and navigated HASTE, the absolute time saving is small and probably out-weighted by lesser image quality.

\section{CARDIOVASCULAR RISK IN ASYMPTOMATIC POTENTIAL SIMULTANEOUS PANCREAS-KIDNEY TRANSPLANT RECIPIENTS IS DETERMINED BY MYOCARDIAL PERFUSION SCINTIGRAPHY}

doi:10.1136/heartjnl-2011-300198.123

V M S Stoll, N S Sabharwal, 00 Ormerod. The John Radcliffe Hospital, Oxford, UK

Introduction More than $50 \%$ of renal transplant recipients will die as a consequence of cardiovascular disease (CVD). Type I diabetics undergoing simultaneous pancreas-kidney transplantation (SPK) are at an even greater risk of CVD. Optimising a patient's cardiovascular status is necessary before SPK transplant surgery. Patients can remain on transplant waiting lists for years. There is little evidence as to how frequently repeat cardiovascular risk assessments are required in asymptomatic patients. Myocardial perfusion scintigraphy is used in SPK patients to detect any asymptomatic myocardial ischaemia or abnormal left ventricular function. This study analyses data from a SPK transplant centre with an annual surveillance programme to aim to establish the suitable frequency of MPS. Methods Potential SPK transplant recipients who had undergone two perfusion scans were included for analysis. An abnormal MPS was defined as either showing a regional wall motion abnormality, inducible ischaemia, or impaired left ventricular function. The scan results were both documented and compared. Angiography results from the study period were also recorded.

Results 99 out of 130 patients on the SPK waiting list in November 2009 had undergone two perfusion scans as part of their 